\title{
Sigmoid Diverticulitis Mimicking Acute Appendicitis in Right-Sided Descending and Sigmoid Colon: A Case Report
}

\author{
Evrim Gül', Yeliz Gül2, Ayşe Nur Gönen³, Zeynep Özkan³, Pınar Gündoğan Bozdağ2, Yılmaz Aslan4 \\ Department of Emergency Medicine, Elazığ Training and Research Hospital, Elazığ, Turkey \\ 2Department of Radiology, Elazığ Training and Research Hospital, Elazı̆̆, Turkey \\ 'Department of General Surgery, Elazığ Training and Research Hospital, Elazı̆̆, Turkey \\ 4Department of Internal Medicine, Elazığ Training and Research Hospital, Elazı̆̆, Turkey
}

\begin{abstract}
Introduction: Acute abdominal pain in the lower-right quadrant can be misdiagnosed as acute appendicitis and may be caused by anatomic variations of the intra-abdominal organs in rare cases. Anatomical variations in the position and morphology of the sigmoid colon should be considered by clinicians and radiologists to avoid misdiagnosis and unnecessary surgical procedures.

Case Report: A 54-year-old man was admitted to our emergency department complaining of abdominal pain in the lowerright quadrant, nausea, vomiting, and fever for 2 days. Physical examination revealed local rebound tenderness on the lowerright quadrant and a temperature of $38^{\circ} \mathrm{C}$. Abdominal ultrasound showed that the intestinal loops were thickened and the surrounding mesenteric tissue was edematous in the right abdominal quadrant. Abdominal computed tomography was performed after a bolus administration of intravenous contrast material, and sigmoid colon position anomalies and diverticulitis were observed. The patient was treated medically, and diverticulosis was confirmed by colonoscopy.

Conclusion: Clinical evaluation and ordered appropriate imaging methods by the emergency specialist is important in patients with abdominal pain on the lower-right quadrant who are admitted to the emergency department. Thus, rare anatomical variations mimicking acute appendicitis can be diagnosed accurately, and patients can be protected from undergoing unnecessary surgeries.
\end{abstract}

Keywords: Position anomaly of colon, diverticulitis, acute appendicitis

Received: 19.12.2015 Accepted: 08.04.2016 Available Online Date: 15.07.2016

\section{Introduction}

Abdominal pain in the lower-right quadrant is usually misdiagnosed as acute appendicitis and may be caused by anatomic variations of the intra-abdominal organs in rare cases. Pain affecting the right iliac fossa is an extremely rare symptom of sigmoid diverticulosis, which is usually associated with pain affecting the left iliac fossa (1, 2). Abdominal computed tomography (CT) is a sensitive imaging modality for detecting sigmoid diverticulosis in older patients with acute pain admitted to an emergency department (3). In this case report, we present a patient with sigmoid diverticulitis mimicking acute appendicitis of the right descending and sigmoid colon that was detected by abdominal CT. The patient's symptoms were successfully treated by antibiotics and parenteral nutrition. Follow-up colonoscopy revealed multiple diverticular orifices in the sigmoid colon.

\section{Case Report}

A 54-year-old man was admitted to our emergency department complaining of abdominal pain in the lower-right quadrant, nausea, vomiting, and fever for 2 days. The patient had not previously undergone abdominal surgery and had no chronic diseases. Physical examination revealed local rebound tenderness over the ileocecal region, a temperature of $38^{\circ} \mathrm{C}$, a heart rate 


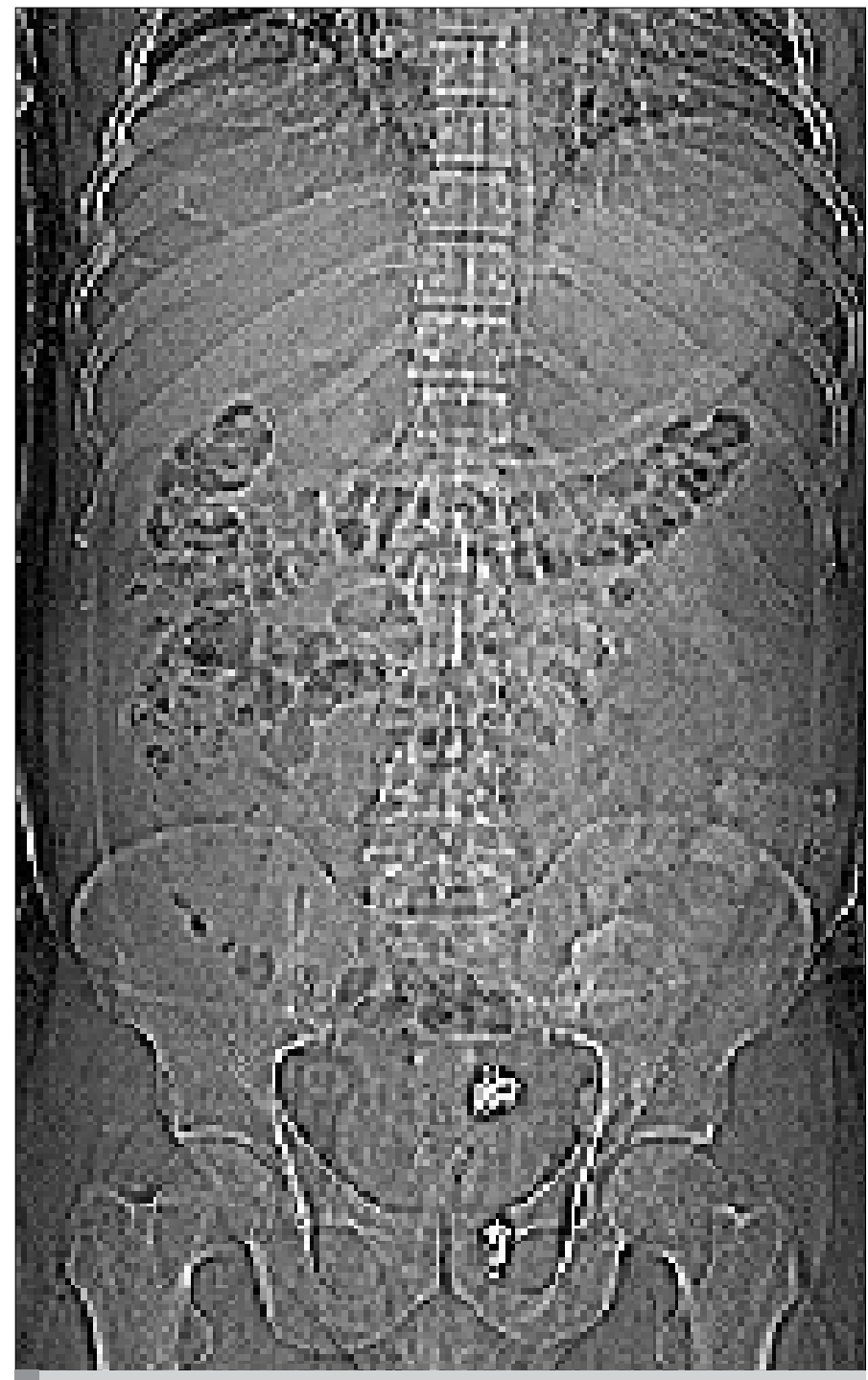

FIGURE 1. Plain abdominal radiography. The radiographic images show the absence of air on the left side and massive air accumulation on the right side

of 88 beats/min, and a blood pressure of 110/70 mmHg. Laboratory findings included a white blood cell count of $11.58 \times 103 / \mathrm{L}$, hemoglobin level of $13.6 \mathrm{mg} / \mathrm{L}$, and normal liver function. Plain abdominal radiography showed that no air was present on the left side, whereas there was a massive accumulation of air on the right side (Figure 1). Abdominal ultrasound (Toshiba Aplio; Tokyo, Japan) of the lower-right quadrant showed that the intestinal loops were thickened and the surrounding mesenteric tissue was edematous. In addition, a 9-mm hyper echogenic region was found on the medial intestine wall, suggestive of appendicolitis, and the right paracolic fossa contained free fluid, suggestive of retrocecal acute appendicitis (Figure 2). The patient was admitted to the General Surgery Clinic with an initial diagnosis of an acute abdomen. Emergency CT (Toshiba asteion 4 scanner; Tokyo, Japan) was performed after a bolus administration of intravenous contrast material. The CT images (Figure 3) showed that the cecum, ascending colon, and transverse colon were normally located. However, after the splenic flexure, the

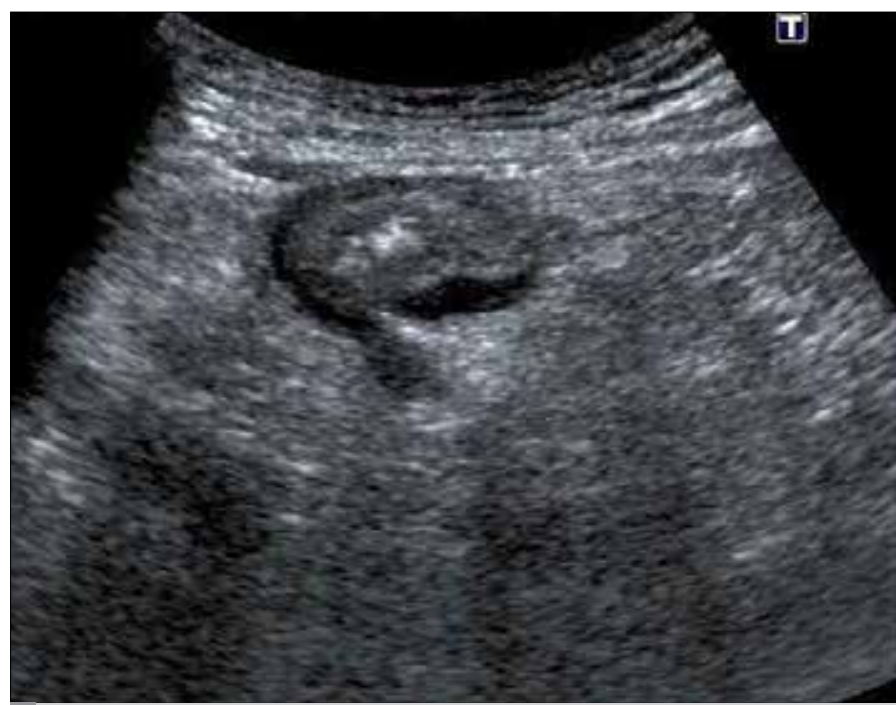

FIGURE 2. Abdominal ultrasound image of right-sided sigmoid diverticulitis mimicking acute appendicitis

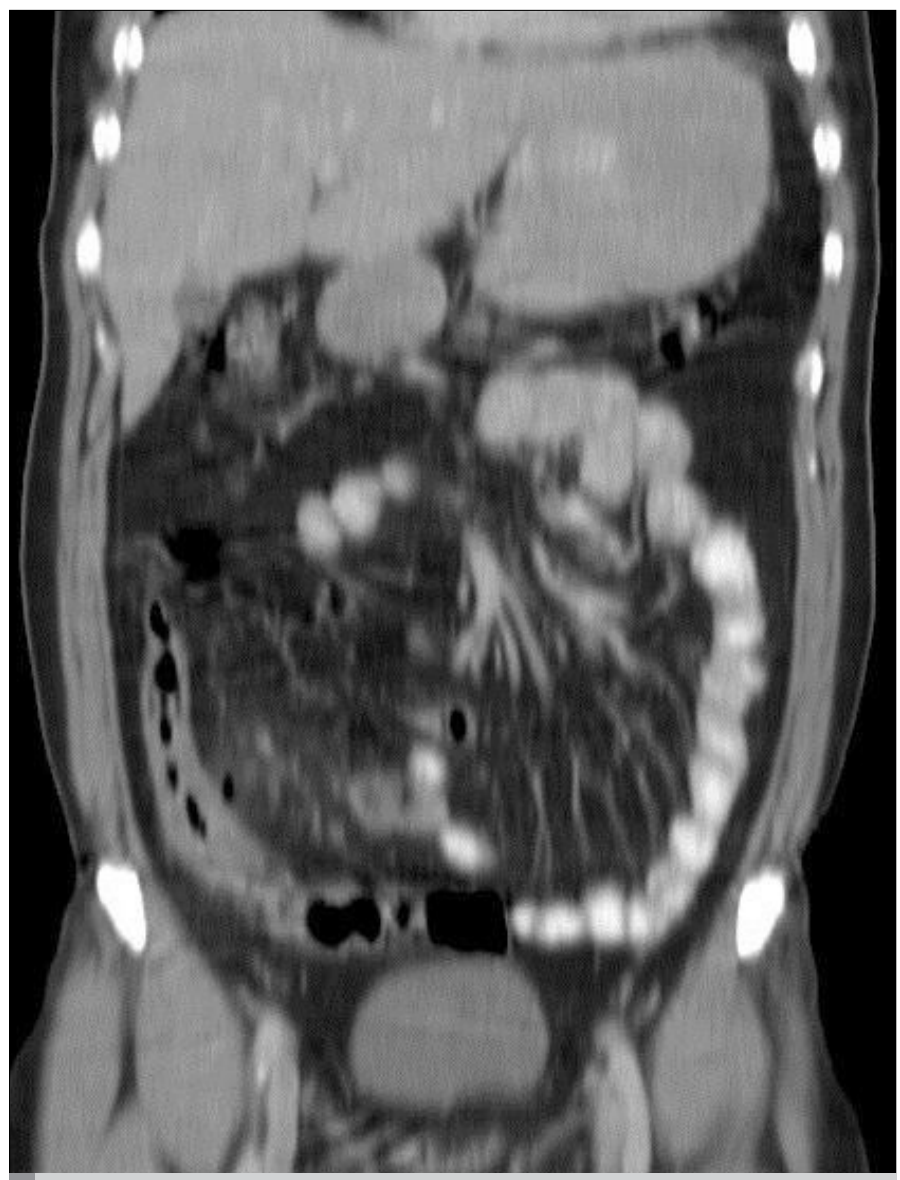

FIGURE 3a. Contrast-enhanced computed tomography (CT). Coronal contrast-enhanced CT image showing congenital anomalies of the descending colon and sigmoid colon. Axial contrast-enhanced CT image showing diffuse wall thickening along the sigmoid colon and increased density of the surrounding mesenteric tissue. In addition, the medial wall of the adjacent sigmoid colon con-tained a several millimeter thick layer of air 


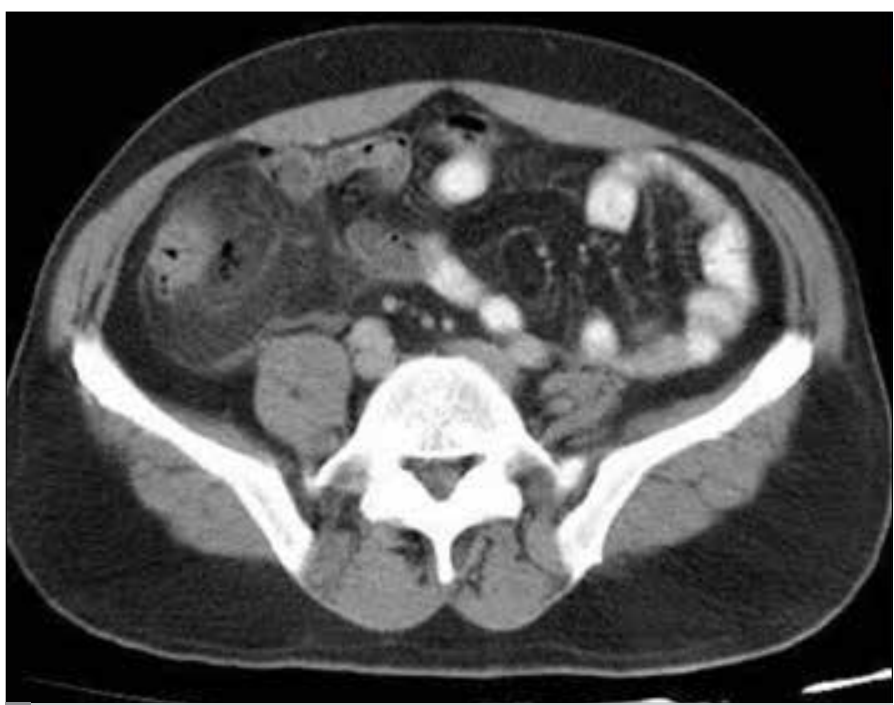

FIGURE 3b. Colonoscopy confirmed the presence of multiple diverticular orifices in the sigmoid colon

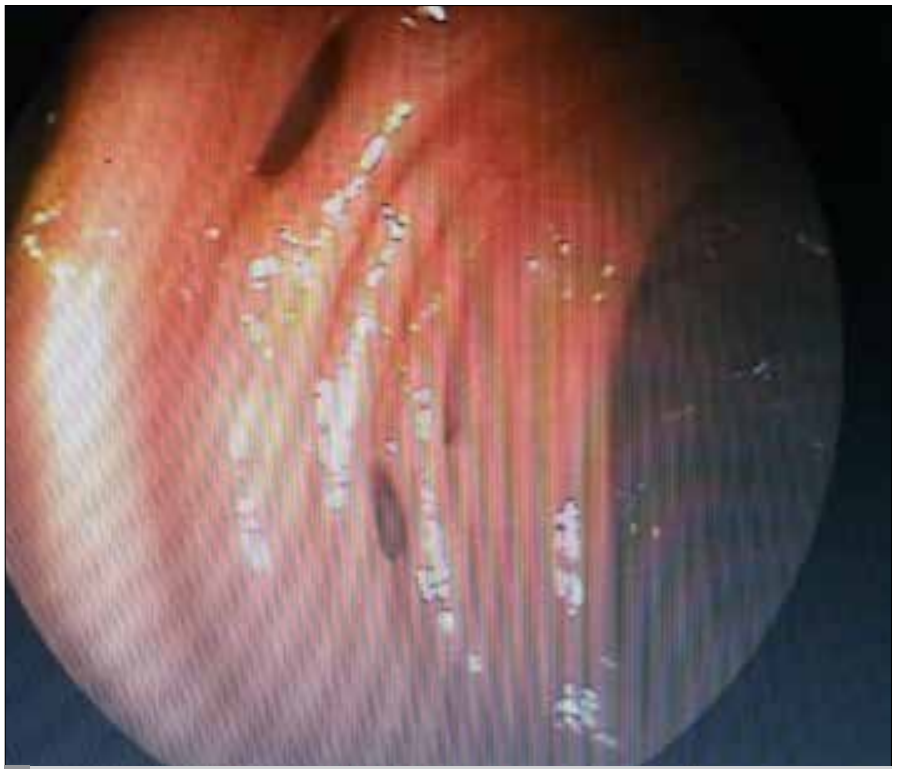

FIGURE 4. Colonoscopy confirmed the presence of multiple diverticular orifices in the sigmoid colon

descending colon returned to the right side toward the midline and the sigmoid colon was directed to the right side. In addition to diffuse wall thickening $(1 \mathrm{~cm})$ of a $10-\mathrm{cm}$ segment of the sigmoid colon, the surrounding mesenteric tissue had an increased density and the medial wall of the adjacent sigmoid colon included a millimetric air density, consistent with a diagnosis of sigmoid diverticulitis with a closed perforation. There was no evidence of a paracolic abscess. The appendix and cecum were normal.

The patient was treated with antibiotics (intravenous ceftriaxone 1 g, twice daily and intravenous metranidazole $750 \mathrm{mg}$ ) and total parenteral nutrition via a peripheral venous catheter. The patient's fever subsided, his leukocyte count decreased, and physical examination was normal. Subsequent ultrasound scans taken at regular intervals showed no signs of an abscess or fluid in the abdomen. Eight days after starting treatment, the patient tolerated oral food and was discharged. Follow-up colonoscopy (Fujinon EPX-4400 System; EG- 530 WL, Tokyo, Japan) 3 months after discharge revealed the presence of multiple diverticular orifices in the sigmoid colon but no other coIonic abnormalities (Figure 4). Informed consent was obtained from the patient.

\section{Discussion}

Congenital anomalies of the intestine, such as rotation and fixation, are generally diagnosed in childhood and are rarely diagnosed in adulthood (2). Srivastava et al. (2) reported an anatomic cadaveric dissection in which the right colon was considered in four sections: the first part turned from the splenic flexure to the bottom right and ended at the level of the number 5 lumbar vertebrae; the second part lay upward and to the right up to the level of the number 2 lumbar vertebrae; the following part extended downward obliquely on the right abdominal side to the pelvic region; and the final part of sigmoid colon originated from the pelvic inlet and lay toward the number 3 sacral vertebrae.

In a cadaver with an excessively long sigmoid colon, Komiyama et al. (4) found that the descending colon formed a loop behind the ascending colon. The first part of the colon was located in the left iliac fossa and extended across the right iliac fossa to the lower abdominal wall. It then followed an upward path to the right lobe of the liver behind the ascending colon, after which it turned backward and descended to the right iliac fossa. The final part followed a medial path and joined the rectum at the median line (4). Fiorella and Donnely (5) reported a right sigmoid colon that was detected radiographically by barium enema. Herein, we describe a case with anatomic variations of the colon that were detected by CT. The anatomic features were similar to the features of the case described by Sirivasta et al (2).

Diverticulosis is commonly seen in Western countries, with sigmoid diverticulosis being one of the most common types $(6,7)$. It was reported that $75 \%$ of patients with diverticulosis showed no symptoms until death, but in $25 \%$ of cases, some complications can occur, such as infection, constipation, diarrhea, hematochezia, abdominal cramps, and colonic obstruction (7). The differential diagnosis of acute abdominal pain should be made using appropriate imaging modalities, including ultrasound and CT. Ultrasound is a quick, easy, and relatively painless imaging technique, when performed by experienced users, to measure the size and content of abscesses. Ultrasound evaluation is a reliable method for the diagnosis of right-sided diverticulitis, with high sensitivity (91.3\%) and specificity $(99.5 \%)(7,8)$.

Abdominal CT is the most beneficial imaging modality for evaluating hollow viscera, such as in the colon, because it provides more information than other imaging methods regarding the location, position, and involvement of adjacent organs or structures in cases of suspected diverticulosis. Although our patient presented with clinical symptoms consistent with acute appendicitis, we could not view the appendix on an ultrasound and there was no air on the left side of the abdomen on plain abdominal radiography. Therefore, we 
decided to perform contrast-enhanced $\mathrm{CT}$ to determine the cause of pain in this patient.

The CT images revealed variations in the anatomic positions of the descending colon and sigmoid colon as well as sigmoid diverticulitis. Unlike acute appendicitis, medical treatment is often sufficient to treat diverticulitis, although some cases might require surgery (8). Medical treatment should be started as soon as possible in the emergency department and should include antibiotics to treat common colonic bacteria, including gram-negative and anaerobic bacteria; intravenous fluid replacement; and analgesics. Medical treatment has a high success rate of $70 \%-100 \%$ for treating patients. Surgical intervention should be performed for usually complicated patients with perforation and bleeding (7).

This case was accurately diagnosed by $C T$ and clinical evaluation and was successfully treated by conservative medical therapy. Unnecessary surgical intervention was also avoided. Three months after discharge, diverticulosis was confirmed by colonoscopy.

\section{Conclusion}

The differential diagnosis of adults presenting with abdominal pain in the lower-right quadrant should include anatomic variations of the gut as well as acute appendicitis. If anatomic variations are suspected, appropriate imaging modalities, including ultrasound and abdominal CT, should be used.

Informed Consent: Written informed consent was obtained from patient who participated in this case.

Peer-review: Externally peer-reviewed.
Author contributions: Concept - Y.G.; Design - Z.Ö., E.G.; Supervision - Z.Ö., Y.A.; Resource - Y.G., A.N.G.; Materials - Y.G., A.N.G.; Data Collection\&/or Processing - P.G.B., Y.G.; Analysis\&/or Interpretation - Y. A., Z.Ö.; Literature Search - E.G.; Writing - E.G., Z.Ö.; Critical Reviews - Y.A., P.G.B.

Acknowledgements: The authors for conributions thank you Dr. Mustafa Uçarel.

Conflict of Interest: The authors declared no conflict of interest.

Financial Disclosure: The authors declared that this study has received no financial support.

\section{References}

1. Anderton M, Griffiths B, Ferguson G. Giant sigmoid diverticulitis mimicking acute appendici-tis. Am R Coll Surg Engl 2011; 93: 89-90. [CrossRef]

2. Srivastava P, Tuli A, Kaur S, Raheja S. Right sided descending and sigmoid colon: its em-bryologi-cal basis and clinical implications. Anat Cell Biol 2013; 46: 299-302. [CrossRef]

3. Hustey FM, Meldon SW, Banet GA, Gerson LW, Blanda M, Lewis LMI. The use of abdominal com-puted tomography in older ED patients with acute abdominal pain. Am J Emerg Med 2005; 23: 259-65. [CrossRef]

4. Komiyama M, Shimada Y. A case of a right-sided sigmoid colon. Kaibogaku Zasshi 1991; 66: 537-40.

5. Fiorella DJ, Donnelly LF. Frequency of right lower quadrantposition of the sigmoid colon in in-fants and young children. Radiology 2001; 219: 91-4. [CrossRef]

6. Salzman H, Lillie D. Diverticular disease: diagnosis and treatment. Am Fam Physician 2005; 72: 1229-34.

7. Bilic Komarica E, Zvizdic Z. Right-sided perforated ascending colonic diverticulum mimicking acute appendicitis. Acta Inform Med 2012; 20: 269-70 [CrossRef]

8. Little A 3rd, Culver A. Right-sided sigmoid diverticular perforation. West J Emerg Med 2012; 13: 103-5. [CrossRef] 